
allemande

47-1| 2015

L'Allemagne et l'Europe | La déprise de l'Empire napoléonien en Allemagne en 1813

\title{
L'intelligentsia littéraire est-allemande et la chute du mur de Berlin : un discours sur soi
}

\section{Carol-Ann Bellefeuille}

\author{
(2) OpenEdition \\ Journals \\ Édition électronique \\ URL : https://journals.openedition.org/allemagne/483 \\ DOI : 10.4000 /allemagne.483 \\ ISSN : 2605-7913 \\ Éditeur \\ Société d'études allemandes \\ Édition imprimée \\ Date de publication : 26 juin 2015 \\ Pagination : 225-241 \\ ISSN : 0035-0974 \\ Référence électronique \\ Carol-Ann Bellefeuille, «L'intelligentsia littéraire est-allemande et la chute du mur de Berlin : un \\ discours sur soi », Revue d'Allemagne et des pays de langue allemande [En ligne], 47-1 | 2015, mis en \\ ligne le 13 décembre 2017, consulté le 21 mai 2021. URL : http://journals.openedition.org/allemagne/ \\ 483 ; DOI : https://doi.org/10.4000/allemagne.483
}




\title{
L'intelligentsia littéraire est-allemande et la chute du mur de Berlin: un discours sur soi
}

\author{
- Carol-Ann Bellefeuille*
}

À l'automne 1989, un mouvement de contestation populaire se met en branle en République démocratique allemande (RDA). Plusieurs facteurs peuvent expliquer ces protestations: la croissance de l'insatisfaction politique des citoyens, notamment à la suite des élections truquées de mai 1989, la libéralisation qui s'exerce en URSS et dans d'autres pays de l'Est alors que la pereströ̈ka de Mikhaïl Gorbatchev fait son chemin, les exils de plus en plus massifs vers la République fédérale allemande (RFA) et, enfin, l'incapacité du Parti socialiste unifié d'Allemagne (Sozialistische Einheitspartei Deutschlands, SED) à répondre adéquatement aux protestations des citoyens $^{(1)}$.

À ce sujet, Günter Schabowski, qui était à l'époque membre du Politburo du SED et qui a prononcé l'ouverture du mur de Berlin le soir du 9 novembre 1989, déclarait en 2009: " Je suis d'avis que nous avons tout fait mal, puisque la tentative de créer une société socialiste est dès le départ vouée à l'échec. L'homme n'est pas en mesure de faire taire son ego. De ce fait, le socialisme est toujours une mauvaise expérience ${ }^{(2)}$. Schabowski était pourtant un acteur important du gouvernement de l'Allemagne de l'Est et, selon ses propres mots, voulait toujours en 1989 sauver la RDA communiste ${ }^{(3)}$. En reconnaissant que le SED n'agissait pas dans l'intérêt de ses citoyens, il arrive néanmoins à s'expliquer l'insatisfaction de la population. Ce mea-culpa n'a toutefois pas été partagé par l'ensemble de l'élite de l'ex-Allemagne de l'Est, et une partie non

\footnotetext{
* Étudiante en maîtrise d'histoire à l'Université de Montréal.

1 À ce sujet, voir notamment Karl Dieter Opp, Peter Voss et Christiane Gern, Origins of a Spontaneous Revolution: East Germany, 1989, Michigan, University of Michigan Press, 1995, p. 43, 176, 190-192.

2 Matthias Schlegel et Christian Tretbar, "Günter Schabowski "Wir wollten uns mit dem Westen arrangieren" ", Die Zeit, 9 novembre 2009. Les traductions d'extraits allemands et anglais sont de l'auteur.

3 AFP, « Meet the man who brought down the Berlin Wall », The Local, 20 septembre 2009.
} 
négligeable de l'intelligentsia a conservé après novembre 1989 un discours résolument socialiste, malgré une participation parfois active dans les manifestations et contestations. C'est le cas d'écrivains parmi les mieux établis de RDA, dont Christa Wolf, Stefan Heym, Christoph Hein, Volker Braun et Heiner Müller ${ }^{(4)}$.

Puisque l'idéologie marxiste-léniniste prévoit l'utilisation de l'élite culturelle dans le but de construire le nouveau peuple socialiste, les écrivains est-allemands jouissaient d'un statut social privilégié en échange de leur loyauté idéologique ${ }^{(5)}$. La littérature constituait ainsi un champ particulier du domaine intellectuel de RDA, ce qui explique qu'il s'y soit développé un discours critique toutefois maintenu à l'intérieur des limites posées par le régime.

Ainsi, à l'automne 1989, devant la montée des protestations et le recul de l'État, les écrivains ont pris la parole afin de réclamer des réformes et se sont déclarés solidaires de la population. Cependant, après l'ouverture du Mur et l'abandon de plus en plus manifeste des idéaux socialistes par le mouvement de contestation, leurs critiques se tournent vers les citoyens désignés comme traîtres. Comment expliquer ce changement de discours? Faut-il donc croire ces écrivains est-allemands lorsqu'ils affirment qu'ils étaient dissidents? Et qu'est-ce qui explique en fait leur réaction négative après la chute du mur de Berlin?

Il est d'abord important de souligner que, lorsque se produisent les premières manifestations à Leipzig en septembre 1989, l'intelligentsia ne joue pratiquement aucun rôle. Il faudra attendre le 4 novembre avant de voir les écrivains et autres artistes est-allemands prendre la rue et participer en tant que groupe à une manifestation. On peut donc croire, comme le sociologue Christian Joppke, que l'élite culturelle est « intervenue seulement lorsque l'existence autonome de l'État a été menacée » ${ }^{(6)}$.

Si l'action est en effet venue des masses citoyennes, c'est plutôt par la parole que les écrivains se sont manifestés. Ainsi, ils se sont prononcés à maintes reprises sur les événements qui se produisaient dans leur pays, par le biais de publications dans les journaux, d'entrevues et de discours publics, visiblement dans l'objectif d'occuper l'avant-scène dans cette contestation qui prenait naissance. On retrouve dans leur discours avant le 9 novembre 1989 une volonté de créer une image positive d'euxmêmes en fonction des revendications et des valeurs portées par la population estallemande. De ce fait, alors que les citoyens se révoltent contre le régime et réclament une liberté plus grande, les écrivains se décrieront comme des victimes de l'État et des dissidents opposés à la ligne idéologique du Parti. Ce faisant, ils espèrent obtenir la position de «porte-paroles » dans cette révolution qui s'entame et affirment parler au nom du peuple pour lequel ils seraient en «mission ». Toutefois, on sent rapidement que leurs volontés s'éloignent des revendications populaires et que leur

4 Nous avons décidé de concentrer notre étude sur les écrits de ces cinq auteurs, puisqu'ils jouissaient d'une tribune importante en RDA, du fait de leur statut. D'ailleurs, on retrouve les idées qu'ils avancent dans plusieurs textes d'autres écrivains est-allemands de l'époque, ce qui témoigne de leur grande influence. 
discours ressemble en fait à celui des politiciens du SED; ils en perdront une grande part de leur autorité. Dans un dernier temps, alors que s'installe de plus en plus l'idée d'unification allemande, les écrivains insisteront sur leur position de victimes face à une RFA capitaliste qui n'apporte aucune aide à la culture. Ce faisant, ils développent une forte critique de la population est-allemande qui se tourne alors vers l'Occident et abandonne le socialisme.

Il semble que l'intelligentsia littéraire, en 1989, souhaitait avant tout se placer à la tête du mouvement de protestation dans le but de le diriger vers des réformes plutôt que vers une révolution dangereuse pour la survie de l'État socialiste. En effet, des écrivains, particulièrement Christa Wolf, Stefan Heym, Christoph Hein, Volker Braun et Heiner Müller, ont pris la parole et participé à des manifestations populaires afin d'user de leur prestige pour conserver une société communiste. Leur réaction négative peut ainsi s'expliquer par un refus de rejeter les quarante dernières années de leur vie au cours desquelles ils s'étaient efforcés de donner un sens à l'utopie socialiste.

\section{Survol de l'historiographie}

Le corpus d'études réalisées au sujet de l'intelligentsia littéraire, de la dissidence et des politiques culturelles de RDA entre 1948 et 1990, sans être abondant, est somme toute étoffé. Les chercheurs ayant travaillé sur ces questions proposent des thèses qui divergent sur deux thèmes primordiaux: la position de l'élite culturelle dans la société et la force de l'État.

Les écrivains sont présentés de trois façons différentes dans l'historiographie. Plusieurs chercheurs, surtout des littéraires et des germanistes, affirment d'abord que ceux-ci étaient victimes, et donc dissidents, d'un pouvoir autoritaire et de sa censure. En fait, ces intellectuels auraient été dupés par le SED qui leur confiait l'éducation du peuple après le nazisme en échange de leur loyauté; on retrouve cette idée chez Dorothea Dornhof et Wilfried van der Will ${ }^{(7)}$. Ces auteurs affirment que, malgré tout, les écrivains n’ont jamais cessé de critiquer le régime. Anne-Marie Corbin va plus loin à ce sujet de la dissidence en déclarant que la répression étatique les aurait menés à développer une opposition profonde au régime, et que «par leurs critiques [et] les alternatives qu'ils proposaient, ils ont préparé le terrain [...] » à la révolution de $1989^{(8)}$. Le germaniste Ian Wallace soutient d'ailleurs qu'ils auraient de ce fait gagné la confiance de la population, jusqu'à ce que cette dernière se détourne des idéaux socialistes $^{(9)}$. Ainsi, si ces chercheurs s'entendent somme toute pour affirmer que l'intelligentsia littéraire profitait effectivement de certains privilèges auxquels la population

7 Dorothea Dornhof, "The Inconsequence of Doubt: Intellectuals and the discourse on socialist unity », in: Michael Geyer (éd.), The Power of Intellectuals in Contemporary Germany, Chicago, University of Chicago Press, 2001, p. 59; Wilfried vAN DER WILL, " The Nature of Dissidence in the GDR », in: Ian WALLACE (éd.), The GDR in the 1980s, Dundee, Loughborough GDR monitor, 1984, p. 41.

8 Anne-Marie Corbin-SCHufells, La force de la parole: les intellectuels face à la RDA et à l'unification allemande, Paris, Presses Universitaires du Septentrion, 1998, p. 243.

9 Ian WALlaCe, "The Failure of GDR Cultural Policy under Honecker ", in: Joachim GlaEssner et Ian Wallace (éd.), The German Revolution of 1989, Oxford, Berg, 1992, p. 113-114. 
n'avait pas accès, ils semblent en fait vouloir la défendre, notamment dans le cadre de certaines controverses soulevées après la chute du Mur ${ }^{(10)}$.

D’autres chercheurs considèrent les écrivains comme un outil utile au SED. Chez Frank Trommler, ces derniers occupent une fonction sociale offerte par l'État-Parti. Le SED aurait en fait été soucieux d'éviter de laisser l'espace public ouvert aux sciences sociales, considérées comme plus susceptibles de déclencher des conflits ${ }^{(11)}$. Toutefois, cette idée n'implique pas de reprocher aux écrivains d'avoir volontairement entretenu une complicité avec le pouvoir, puisqu'ils évoluaient dans une société dictatoriale qui ne leur laissait pas le loisir de choisir leur position. La disparition de la RDA aurait ainsi laissé l'intelligentsia littéraire sans fonction sociale, ce qui serait la cause de sa réaction négative en 1990. Peter C. Pfeiffer ajoute que c'est en fait l'idéal antifasciste des écrivains qui les aurait menés à légitimer involontairement le régime ${ }^{(12)}$ : puisque le SED avait travaillé à faire de la RDA l'incarnation de cet antifascisme auquel les écrivains tenaient plus qu'à toute autre idée, il aurait été impossible pour eux d'accepter sa disparition. Ces intellectuels participaient ainsi à leur insu à stabiliser l'État, et même leurs critiques, selon Konrad Jarausch, étaient instrumentalisées en ce sens par le régime qui les tolérait dans le but de laisser croire à une société libre ${ }^{(13)}$.

La sincérité, ou l'innocence, des écrivains est remise en doute par un dernier groupe de chercheurs, dont Simone Barck, Martina Langermann et Siegfried Lokatis. Ces auteurs acceptent la conception selon laquelle la littérature était victime de censure, mais ils soulignent que ses acteurs étaient tout de même peu enthousiastes à l'idée d'offrir la parole aux autres citoyens ${ }^{(14)}$. En ce sens, Sara Jones écrit: " chaque groupe [les écrivains et le régime] avait besoin de l'autre [...] " ${ }^{(15)}$. Elle soutient que les écrivains auraient avant tout voulu créer un espace de négociation dans les coulisses du régime afin de contourner les limites de la censure, cette complicité étant nécessaire à leur carrière. La dissidence d'un auteur comme Stefan Heym aurait de ce fait été utilisée en tant que levier pour mettre de la pression sur un État qui craignait de voir son image écorchée. À ce sujet, Christian Joppke insiste sur le fait qu'en 1989, les écrivains ne réclamaient que des réformes et surtout la sauvegarde de l'État socialiste, alors que les mouvements populaires entamaient une révolution. Selon lui, la véritable dissidence a donc été citoyenne, les intellectuels conservant des revendications foncièrement socialistes. Wolfgang Emmerich, dans le même ordre d'idées, affirme que les écrivains se

10 On pense ici à la controverse soulevée par la publication de Was bleibt de Christa Wolf en 1989 et aux polémiques déclenchées par l'ouverture des dossiers de la Stasi dès 1992 qui ont dévoilé la collaboration de plusieurs écrivains avec l'État policier. À ce sujet, voir Sara Jones, Complicity, Censorship and Criticism: Negotiating Space in the GDR Literary Sphere, Berlin, Éditions de Gruyter, 2011, p. 37-39.

11 Frank Trommler, "German Intellectuals: Public Roles and the Rise of Therapeutic ", in: M. Geyer (éd.), The Power of Intellectuals (note 7), p. 54.

12 Peter C. Pfeiffer, "The national identity of the GDR », in: Friederike Eigler et Peter C. Pfeiffer (éd.), Cultural transformations in the new Germany, Columbia, Camden House, 1993, p. 24-25.

13 Konrad Jarausch, "The Double Disappointment: Revolution, Unification and German Intellectuals », in: M. Geyer (éd.), The Power of Intellectuals (note 7), p. 280.

14 Simone Barck, Martina Langermann et Siegfried Lokatis, «The GDR as a Reading-Nation: Utopia, Planning Reality and Ideology », in: ibid., p. 93.

15 S. Jones, Complicity, Censorship and Criticism (note 10), p. 12. 
seraient en fait eux-mêmes promus au rang d'avant-gardes et de porte-paroles de la population, puisqu'ils vivaient avant tout « avec le désir d'être utiles à la société, d'y être nécessaires, et de participer à leur façon à l'établissement du socialisme » ${ }^{(16)}$.

Pour ce qui est de la puissance de l'État, l'historiographie, tout comme au sujet du rôle social des intellectuels, ne présente pas de consensus : l'État est chez certains chercheurs fort et complètement oppressif, alors qu'il est, chez d'autres, faible, ou affaibli. Cette question de la force du régime est essentielle, car « elle touche à la responsabilité, voire à la culpabilité de chacun des anciens citoyens de la RDA » ${ }^{(17)}$. En effet, le niveau de contrôle déployé par l'État peut être utilisé pour justifier la position compromettante qu'ont pu occuper certains individus sous le régime socialiste. C'est ce qu'on retrouve d'ailleurs dans le discours de certains écrivains dès 1990.

Ainsi, chez des chercheurs tels que Dornhof, Pfeiffer, Trommler et van der Will, qui reconnaissent une position de "victime » aux écrivains de l'ex-RDA, on retrouve une conception rigide du régime, qui contrôlait totalement et oppressait sévèrement les intellectuels en conservant une emprise ferme sur la production culturelle. Sigrid Meuschel abonde dans le même sens lorsqu'elle affirme que le Parti avait relativement réussi à homogénéiser sa société, empêchant ainsi le développement de groupes d'intérêts divergents ${ }^{(18)}$. Matt Killingsworth propose une conception de l'État est-allemand encore plus rigide en soutenant qu'il y existait une "sphère publique totalitaire " ${ }^{(19)} \mathrm{au}$ sein de laquelle le contrôle étatique était si complet que la création de tout discours dissident s'en trouvait freinée. Les intellectuels n'ont donc pas pu s'opposer efficacement au régime, et ce même s'ils le désiraient. Selon Christian Joppke, c'est par un habile mélange de répression et de tolérance arbitraire que l'État réussissait à éviter l'éclosion de dissidence.

Cependant, la thèse de Joppke diffère de celle des auteurs précédemment cités lorsqu'il reconnaît que le mouvement d'exil qui s'est affirmé à l'automne 1989 a fait perdre la possibilité de contrôle absolu au régime et que l'État s'est ainsi tardivement affaibli. Certains auteurs considèrent toutefois que la RDA était un État faible depuis bien plus longtemps. On retrouve cette idée chez des chercheurs qui ne s'entendent pourtant pas sur la fonction sociale de l'intelligentsia. En fait, ce qui éloigne les auteurs considérant les écrivains comme des dissidents de ceux les percevant comme des complices, c'est leur perception de l'usage qui a été fait de cet espace accordé par la faiblesse de l'État. Ainsi, pour Anne-Marie Corbin et Ian Wallace, la tentative d'instrumentalisation des écrivains par le Parti aurait été un échec, puisque cela aurait en fait exacerbé la critique exprimée par ces intellectuels subissant une répression. L'État n'a donc pas su se protéger et a été naïf. Andrew Evans affirme ainsi qu'en voulant jouir de

16 W. Emmerich, « Between Hypertrophy and Melancholy» (note 5), p. 281.

17 Reiner Marcowitz, «Vingt ans après: les années 1989-1990 vues par les historiens », in: Chantal Metzger (dir.), La République démocratique allemande: la vitrine du socialisme et l'envers du miroir, Bruxelles, P.I.E. Peter Lang, 2010, p. 351.

18 Sigrid Meuschel, «Revolution in a Classless Society », in: Glaessner/Wallace (éd.), The German Revolution of 1989 (note 9), p. 144.

19 Matt Killingsworth, Civil Society in Communist Eastern Europe, Colchester, European Consortium for Political Research Press, 2012, p. 80, 104. Bien que l'État n'ait pas eu la mainmise sur toutes les organisations publiques, il y était profondément infiltré et arrivait ainsi à atomiser la société. 
la tribune des intellectuels auprès du peuple, le régime aurait offert à ces écrivains un espace public de liberté, « les implications à long terme étant considérablement plus dommageables que les décideurs ne l'avaient anticipé " ${ }^{(20)}$.

De son côté, Sara Jones n'adhère pas à cette conception de l'écrivain dissident, mais on trouve dans sa thèse le même espace public créé par une ambiguïté ou un affaiblissement dans la position du Parti. Ainsi, il existait au sein de l'État « une tension entre le désir de limiter la disponibilité des ouvrages d'auteurs critiques, et, en même temps, celui de [les] garder intégrés au système de production culturelle ${ }^{(21)}$. Cela aurait rendu le système de publication arbitraire: plus un auteur menaçait de façon crédible d'entacher l'image de la RDA, plus on était enclin à accepter de produire ses textes dont les idées pouvaient pourtant être éloignées du discours étatique. Cela aurait participé à fragmenter l'espace public. Selon Jones, cette tribune a cependant été utilisée par les écrivains pour obtenir des privilèges professionnels.

\section{Un discours sur soi}

Ces différences historiographiques représentent en fait des divergences d'interprétation concernant l'aura de vérité à accorder au discours des écrivains. Certains chercheurs acceptent le postulat émis par leurs propres sujets de recherche et utilisent le discours de l'intelligentsia littéraire en tant que source pour définir le rôle social de ces individus. Il semble pourtant nécessaire d'interpréter de façon critique ce discours, puisque ses émetteurs étaient avant tout des êtres intéressés.

Puisqu'on a reproché à plusieurs écrivains d'avoir profité de privilèges importants au sein du régime socialiste et puisque l'ouverture des dossiers de la Stasi a dévoilé que certains d'entre eux ont collaboré dans une certaine mesure avec l'État policier ${ }^{(22)}$, on retrouve dans leurs publications longtemps après la chute du Mur une volonté de se dépeindre en tant que victimes et dissidents en RDA. Il semble qu'ils aient de cette façon voulu contrer l'image de collaborateurs qu'on leur attribuait afin de légitimer leur discours sur le socialisme. Aucun diagnostic ne sera fait ici au sujet de la validité ou non de cette image de complicité qui a été accolée à certains intellectuels après 1989. L'objectif est plutôt d'exposer de quelle manière et en quels termes les écrivains ont tenté de donner de la légitimité à leur discours en 1989 et 1990, et de déceler les ambiguïtés qui en ont découlé.

20 Andrew Evans, "The Last Gasp of Socialism: Economics and Culture in 1960s East Germany ", German Life and Letters, 63/3 (2010), p. 342.

21 S. Jones, Complicity, Censorship and Criticism (note 10), p. 129.

22 Ces privilèges s'expliquent notamment par l'idéologie marxiste-léniniste, qui prévoit utiliser l'élite intellectuelle et culturelle pour modeler le « nouvel homme socialiste ». Cela offre un statut privilégié à l'intelligentsia, mais implique aussi qu'elle doive afficher sa loyauté au régime. À ce sujet, voir W. Emmerich, «Between Hypertrophy and Melancholy» (note 5), p. 277; voir aussi Anne-Marie Corbin-Schufells, La force de la parole: les intellectuels face à la RDA et à l'unification allemande, Paris, Presses universitaires du Septentrion, 1998, p. 202. 


\section{Victimes du régime}

La première position que revendiquent les écrivains est celle de victime du régime, en insistant sur la censure et l'oppression étatique dont ils faisaient l'objet. Cette thématique est particulièrement présente chez Christa Wolf, qui publie en 1990 un récit semi-autobiographique, Was bleibt (Ce qui reste), dans lequel elle dépeint la surveillance qu'elle subissait et la peur que cela éveillait chez elle. Décrivant sa constante position de méfiance, Wolf semble souhaiter que le lecteur sente à quel point le régime nuisait à son art et à sa vie. Elle affirme ainsi s'être sentie impuissante à faire quoi que ce soit dans cet État répressif: "Parler c'est impossible, mais je ne veux pas me taire non plus... notre vie est sans alternative ${ }^{(23)}$.

La censure et l'oppression exercée par le régime ne peuvent être mises en doute. L'appareil étatique de contrôle des publications était effectivement important et le processus de censure très développé. De plus, les écrivains subissaient une pression les poussant à l'autocensure et l'Union des écrivains de RDA jouait un rôle de premier plan à ce sujet, puisqu'il fallait en faire partie afin d'être officiellement reconnu par le régime ${ }^{(24)}$. Ainsi, les quelques figures importantes de la littérature est-allemande qui en ont été exclues au cours des années, dont Stefan Heym et Heiner Müller, peuvent jusqu'à un certain point être considérées comme des victimes. Cependant, il est important de souligner que cela ne les a pas empêchées, dans l'absolu, de continuer à produire et à publier des œuvres.

D’ailleurs, le cas de Stefan Heym est significatif: cet écrivain résolument socialiste s'est somme toute maintes fois brouillé avec le Parti qui le considérait trop critique. Toutefois, c'est parce que le SED le jugeait justement critique qu'il lui permettait de voyager à l'Ouest et que Heym trouvait toujours une façon de se faire publier - malgré des difficultés administratives périodiques. En fait, c'est par la menace de ternir l'image de la RDA à l'étranger que cet auteur arrivait à créer un espace de négociation dans les coulisses du régime afin d'obtenir des avantages pour sa propre carrière et sa propre liberté d'expression ${ }^{(25)}$. Difficile d'y voir une victime passive soumise à la volonté du régime.

Il semble ainsi pertinent de remettre en question la façon dont a été décrite cette victimisation par les écrivains dès 1989. On constate qu'ils insistent particulièrement sur ce rôle de victime, en laissant dans l'ombre les privilèges dont ils bénéficiaient pourtant. Ainsi, encore une fois dans Was bleibt, Christa Wolf met l'accent sur la peur qui l'occupe alors que des policiers de la Stasi surveillent son appartement, mais elle ne soulève à aucun moment le fait que le niveau de vie du "personnage » qu'elle décrit est somme toute plus élevé que celui des citoyens ordinaires ${ }^{(26)}$. On peut aussi se questionner sur les intentions de l'auteur qui choisit de publier ce texte en 1990, une fois le

23 Bettina PARrY, «Christa Wolf: ils ne m'auront pas », l’Humanité, 12 janvier 1996.

24 À ce sujet, voir BARCK/LANGERMANN/LokATIs, "The GDR as a Reading-Nation » (note 14), p. 95-100, et S. Jones, Complicity, Censorship and Criticism (note 10), p. 77.

25 Il s'agit de la thèse défendue par Sara Jones, appuyée sur ses recherches dans les archives du SED.

26 Christa Wolf, Ce qui reste, Aix-en-Provence, Alinea, 1990, 103 p. C'est un personnage "fictif " que présente Wolf: une femme, auteur, qui participe à plusieurs rassemblements culturels et est surveillée par la Stasi. Cela ressemble en tout point à Wolf elle-même. 
régime autoritaire défait, alors qu'elle avait commencé à l'écrire en 1979. De nombreux détracteurs ont relevé ce point lors de la publication du livre, se demandant si Wolf ne cherchait pas en fait à purifier son image alors que, dans la réalité, elle aurait été privilégiée par l'État tombé en disgrâce.

Il a bien été démontré que les écrivains jouissaient d'avantages auxquels n'avait pas accès l'ensemble de la population. Ils profitaient entre autres d'avantages fiscaux substantiels et les mieux établis profitaient d'un confort matériel supérieur, comme l'indique cette déclaration d'Otto Grotewohl, président du Conseil des ministres de RDA entre 1949 et 1964: «La déduction supplémentaire de $40 \%$ des frais professionnels représente une mesure en faveur des intellectuels [...]. Cette mesure démontre quelle importance est attachée aux tâches d'écrivains que fournissent nos intellectuels [...] " ${ }^{(27)}$. Ces avantages peuvent s'expliquer par le fait que « la RDA a toujours accordé à la culture [...] une importance politique énorme, la percevant, tels Jekyll et Hyde, d'un côté comme l'apôtre du type de socialisme promu par le SED et, de l'autre, comme son principal agent de subversion " ${ }^{(28)}$. Ainsi, il importait pour le régime de développer une certaine loyauté chez l'élite culturelle afin d'éviter qu'elle ne se retourne contre lui. Les écrivains avaient donc un statut social privilégié, leur offrant du même coup une tribune influente. Cela est particulièrement vrai pour ceux qui possédaient un statut international, tels Stefan Heym, Christa Wolf et Heiner Müller. Wolfgang Emmerich souligne, dans le même ordre d'idées, que la fonction positive accordée aux intellectuels exerçait un attrait significatif chez les écrivains, les liant d'autant plus au projet socialiste du régime.

De ces privilèges, toutefois, les écrivains ne soufflent mot. Ainsi, dans un autre texte publié après la réunification, Wolf se remémore plutôt les moments difficiles vécus sous la RDA et atteste que les intellectuels en conflit avec le Parti vivaient des crises psychosomatiques qui les atteignaient tant physiquement que psychologiquement ${ }^{(29)}$. De plus, Christoph Hein et Stefan Heym soulèvent le sujet de la censure qui était orchestrée contre eux, l'opposant à leur courage et à leur capacité de se lever contre ce régime pour arriver finalement à être publiés ${ }^{(30)}$. Non seulement ces écrivains se décrivent-ils en tant que victimes, mais ils développent aussi l'idée selon laquelle ils ne se seraient pas laissés abattre par leur sort. De ce fait, Christa Wolf déclare que, lorsque la révolution se met en branle en 1989, les intellectuels se sont libérés de l'État-SED et ont réclamé « ce que l'on [leur] avait pris: le droit d'exprimer librement une opinion critique » ${ }^{(31)}$. Ainsi, c'est d'abord parce qu'ils étaient victimes qu'ils ne pouvaient se révolter contre le régime. Cependant, après septembre 1989, ils affirment qu'ils ont toujours été dissidents et que c'est pourquoi ils peuvent réclamer la fonction d'avantgarde dans la Wende (" le tournant», surnom du mouvement de réformes de 1989 en RDA). Il va sans dire que cette image de «victime » ne pouvant s'exprimer de peur

27 Cité dans A.-M. Corbin-Schufells, La force de la parole (note 22), p. 56.

28 I. WAllace, « The Failure of GDR Cultural Policy» (note 9), p. 102.

29 Christa Wolf, « Voyage d'hiver », in: Ch. Wolf, Ici même, autre part, Paris, Fayard, 1996, p. 67.

30 Claude PrÉvost, « Le serpent et le hérisson », l’Humanité, 16 mars 1990.

31 Christa Wolf, « Mince est la couche de la civilisation », in: Ch. Wolf, Ici même, autre part, p. 183. 
d'être réprimée s'accorde mal avec celle de « dissident " protégeant les intérêts de la population. Pourtant, les écrivains se sont revendiqués des deux.

\section{Dissidence}

Le journaliste du quotidien communiste français l'Humanité, Claude Prévost, écrit en 1989: "Les bouleversements en cours ne surprendront pas tout à fait les familiers de la littérature qui paraissait en RDA ou qui aurait dû y paraître. Les écrivains sont des guetteurs qui perçoivent des signes imperceptibles " ${ }^{(32)}$; ce à quoi son collègue Jean-Paul Piérot ajoute: "Les artistes et les écrivains ont été, dès le début, à la pointe du mouvement révolutionnaire ${ }^{(33)}$. Ces affirmations sont alimentées par les publications et discours de plusieurs écrivains de RDA qui ont alors cherché à « se promouvoir eux-mêmes au rang d'avant-garde conceptuelle " ${ }^{(34)}$ en adaptant leur image au profil de dissident souhaité par le mouvement de revendications populaires.

Plusieurs reviennent donc sur des actions passées qui peuvent, en 1989, prouver leur dissension « de toujours » envers le régime. De nombreuses allusions sont alors faites à l'expatriation de Wolf Biermann en $1976^{(35)}$ : à l'époque, une douzaine de membres de l'Union des écrivains de RDA avaient signé une lettre demandant une plus grande tolérance envers le chansonnier forcé à l'exil. Les écrivains réutilisent cet événement dès 1989 pour démontrer que leur appui d'alors à Biermann représente une dissidence profonde. Cependant, lorsqu'on lit cette lettre, force est de constater qu'ils y étaient demeurés absolument polis et soucieux de leur propre sécurité:

« Nous ne nous identifions pas avec chacun des termes utilisés par Biermann, ni avec chacune de ses actions et nous nous distançons des tentatives de récupération, à des fins nuisibles à la RDA, de la situation créée autour de Biermann [...]. Nous protestons contre sa déchéance de la citoyenneté est-allemande et demandons que les mesures prises à son encontre soient reconsidérées " ${ }^{(36)}$.

Soulignons aussi que cette lettre n'a pas donné lieu au rapatriement du chansonnier et que les signataires n'ont toutefois pas jugé bon de pousser la protestation plus loin.

Il ne s’agit pas du seul événement alors récupéré. En fait, plusieurs mobilisent leur réussite passée à faire publier des textes qui ne cadraient pas totalement avec les canons culturels socialistes pour se présenter comme des critiques acerbes du régime. Ainsi, en mai 1995, peut-on lire dans l'Humanité: "Christoph Hein fut, avec Christa Wolf, Stephan Hermlin et quelques autres, de ceux qui ouvrirent talentueusement la littérature de la RDA à l'universel, faisant obstinément vivre l'esprit critique contre les raideurs dogmatiques et les opportunistes ${ }^{(37)}$. Dans le même ordre d'idées, Heiner

32 Claude Prévost, «Les voyageurs blessés », l’Humanité, 22 novembre 1989, p. 24.

33 Jean-Paul Piérot, "Solidarité Paris-Berlin », l’Humanité, 16 décembre 1989, p. 14.

34 W. Emmerich, « Between Hypertrophy and Melancholy» (note 5), p. 278.

35 Biermann, chansonnier est-allemand très critique du pouvoir, est forcé à l'exil après avoir offert un concert à Cologne en 1976. À ce sujet, voir Anne-Marie CoRBIn, «Rudolf Bahro et Wolf Biermann: deux critiques bien médiatisées du régime de la RDA », in: Ch. MetzGer (dir.), La République démocratique allemande (note 17), p. 59-75.

36 Cité dans ibid., p. 68.

37 Jean-Claude Lebrun, "Chronique de la difficulté des plaines », l'Humanité, 26 mai 1995. 
Müller déclare un peu avant sa mort qu'il n’a jamais songé à quitter la République démocratique allemande, malgré la censure, car il « [avait] besoin de cette confrontation et de ce contexte politique " ${ }^{(38)}$ pour écrire. Ces écrivains insistent donc sur leur profil de dissidents en arguant que leur littérature demeurait libre. Cela permet à Christa Wolf de s'adresser à la population lors de la manifestation organisée par les artistes le 4 novembre 1989 en ces termes: «[...] nous sommes étonnés de nous entendre dire ce que nous pensions depuis longtemps déjà [...] : démocratie maintenant ou jamais! » ${ }^{(39)}$ De même, Stefan Heym se permet d'affirmer que les citoyens « oublient [qu'il] leur [a] préparé la voie en étant le seul à exprimer vingt ans plus tôt ce qu'ils ont enfin osé exprimer en $1989{ }^{(40)}$.

Les intellectuels se posent donc comme des avant-gardistes dans la critique du régime sans prendre en considération que l'État tolérait peut-être leurs écrits idéologiquement déviants en tant que "valve de sûreté » : cette notion, introduite par Konrad Jarausch, suggère que le régime utilisait en fait les publications littéraires critiques pour permettre l'illusion de "normalité » et de liberté de parole au sein de la dictature. D’ailleurs, la littérature critique demeurait foncièrement apolitique et n'attaquait jamais les fondements de l'État. De ce fait, il est difficile de considérer l'action des intellectuels comme de la dissidence si l'on prend en considération que le régime n'en a été à aucun moment menacé. En fait, les individus qui menaçaient réellement l'État ont été, comme Biermann, expatriés ou se sont exilés ${ }^{(41)}$.

Christa Wolf en remet quelques années plus tard en présentant sa génération d'écrivains comme des dissidents à la fois naïfs et désillusionnés:

"Ma génération qui, fière de son inexpérience et tellement assurée de connaître cette communauté humaine amicale pour laquelle nous nous engagions, n'échapperait pas au verdict elle non plus, et nous serions entraînés dans le naufrage de cette expérience en laquelle nous avions arrêté de croire depuis longtemps ${ }^{(42)}$.

Wolf soutient ainsi que la dissidence des écrivains est profonde et remonte à un temps lointain. De plus, elle introduit l'idée, déjà émise par Heym, selon laquelle la population ne reconnaît pas adéquatement l'action dissidente entreprise par l'intelligentsia littéraire.

La manifestation du 4 novembre 1989 sur l'Alexanderplatz est aussi un événement qui sera maintes fois réutilisé dans les discours des intellectuels afin de justifier leur position de tête dans la révolution. Martina Doering, qui était alors journaliste, affirme ainsi que cette journée a été « certainement la plus importante manifestation spontanée depuis la création de cette république [...], le jour du tournant véritable » ${ }^{(43)}$. Le terme «spontanée » ici utilisé peut cependant sembler paradoxal, puisque

38 Cité dans Brigitte Pätzold, « Heiner Müller: la mort d'un homme de théâtre que l'Allemagne réunifiée n’a plus inspiré », l'Humanité, 19 janvier 1996.

39 Christa Wolf, «Citoyen, éteins la télé », in: Nicole BARY, Chronique d’un automne allemand: RDA, 1989 (documents), Paris, La Nuée Bleue et J.C. Lattès, 1990, p. 107. Souligné par l'auteur.

40 Cité dans Brigitte Pätzold, «Stefan Heym: une conscience du siècle », l'Humanité, 18 décembre 2001.

41 On pense notamment à Jürgen Fuchs, Thomas Brasch et Siegmar Faust.

42 Christa Wolf, « Rencontre sur la $3^{\mathrm{e}}$ rue », in: Ch. Wolf, Ici même, autre part, p. 18.

43 Martina Doering, "L'adieu à un pays », Les Temps Modernes, juillet 1990, p. 3. 
la manifestation était autorisée par l'État, qu'un important service d'ordre avait été prévu et que des dirigeants du Parti, dont Günter Schabowski, y ont pris la parole aux côtés des artistes ${ }^{(44)}$.

C'est d'ailleurs à ce moment que la population commence à prendre conscience que le discours de l'intelligentsia culturelle se rapproche en fait sérieusement de celui de l'élite politique socialiste. Les termes utilisés par les deux groupes se ressemblent et les valeurs auxquelles ils font appel sont les mêmes. À l'époque, le Parti tentait aussi de redresser la situation dans le but d'éviter que le mécontentement populaire ne fasse tomber l'État. On promettait ainsi des réformes qui auraient pour but de transformer le socialisme stalinien en un socialisme plus humain. Egon Krenz, devenu secrétaire général du SED après la destitution d'Erich Honecker en octobre 1989, déclare: «En RDA [se produit un] soulèvement social pour un socialisme rénové [...]. Nous n'avons pas besoin de tout reprendre à zéro pour mettre en chantier notre stratégie pour la société [...]. Remettre en question ces bases, ce serait vouloir le chaos, la déstabilisation ${ }^{(45)}$. Il promet ainsi une rénovation du communisme en Allemagne de l'Est et met en garde contre l'institution d'un modèle de société complètement différent. Ces thèmes sont repris par les écrivains: Stefan Heym affirme que «l'échec du socialisme réel, non seulement en RDA mais aussi en Union soviétique, nous permet aujourd'hui d'envisager le socialisme véritable. [...] Le capitalisme n'est pas en mesure de créer un nouvel ordre véritablement humain ${ }^{(46)}$. De son côté, Christoph Hein réclame "un programme qui fasse que cette situation réellement dangereuse disparaisse. Car la menace existe d'une terrible déstabilisation de l'État " ${ }^{(47)}$. Enfin, Volker Braun affirme craindre que « la déception à l'égard du socialisme d'imprégnation stalinienne [soit] si grande qu'on retourne à un point zéro, qui risque de voir jeter l'enfant avec l'eau du bain ${ }^{(48)}$. Il s'agit des mêmes thèmes: danger de déstabilisation de l'État, rénovation du socialisme et rejet du modèle occidental.

Aussi significative est la publication du manifeste Für unser Land (Pour notre pays) le 26 novembre 1989. Ce texte est rédigé par Christa Wolf et signé par plusieurs membres de l'intelligentsia littéraire, dont Volker Braun et Stefan Heym, ainsi que d'autres représentants de l'élite culturelle. Il se lit comme un appel au peuple:

« Nous avons le choix: soit nous tentons de conserver l'autonomie de la RDA [...] en travaillant ensemble, avec les groupes étatiques et les groupes d'intérêts, pour développer une société solidaire respectueuse de la liberté et des droits sociaux, soit nous devrons tolérer, contraints par des considérations économiques, l'influence des riches économistes et politiciens de la RFA et la liquidation de nos valeurs matérielles et morales. Laisseznous prendre la première voie. Nous avons encore la chance de développer une alternative socialiste à la RFA » ${ }^{(49)}$.

44 Claude Marchand, «Parler ouvertement », l'Humanité, 4 novembre 1989, p. 14.

45 Cité dans Claude MARCHAND, «Socialisme à rénover », l'Humanité, 10 novembre 1989, p. 2.

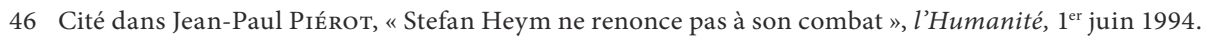

47 Cité dans Gilbert BADIA, «Intellectuels de RDA: l'esprit de Rosa », Révolution, 24 novembre 1989, p. 39.

48 Cité dans Francis Cомве, « Rencontre avec Volker Braun: le dialogue de Hinze et Kunze dans la rue », l'Humanité, 2 novembre 1989, p. 15.

49 Christa Wolf, Für unser Land, 26 novembre 1989. 
Ainsi, ces écrivains refusent d'envisager la dissolution de la RDA socialiste. Le plus grand paradoxe ici relève sûrement du fait qu'Egon Krenz, dans un article publié dans le Neues Deutschland du 30 novembre, donne son approbation au texte et y appose sa signature ${ }^{(50)}$. Les initiateurs du manifeste ne sont évidemment pas enchantés par cet appui du secrétaire général du SED. Il n’en demeure pas moins que cela démontre l'adéquation entre le discours des écrivains et celui du Parti.

Il n'est toutefois pas question pour eux de voir une ambiguïté dans cette ressemblance. Les écrivains continuent à réclamer des réformes pour le socialisme, et justifient leur droit à le faire en insistant sur leur dissidence passée. Selon Christoph Hein, elle remonte aux années 1970, alors que «beaucoup de gens, rompant le silence, n'ont plus accepté que l'on expulse et expatrie les intellectuels et les artistes qui refusaient la discipline ", et que, bien qu' "en RDA [...] il y [eût] une posture intellectuelle souhaitée par l'État », celle-ci " déboucha sur des résultats opposés [...], il était tout à fait normal que des intellectuels aient à cet égard une réaction critique: cela fait partie de leur travail " ${ }^{(51)}$. Pour Hein, être dissident est de ce fait inscrit dans la fonction même de l'intellectuel. L'intelligentsia culturelle affirme de plus ne pas avoir été dupe. C'est ce que veut vraisemblablement exprimer Heiner Müller lorsqu'il affirme: « je pense avoir vécu sans utopie depuis $1961{ }^{(52)}$. On est alors en droit de se demander pourquoi ces écrivains sont demeurés en RDA, s'ils y étaient à la fois victimes et opposants. À cela, ils répondent par la fabrication d'une identité « missionnaire ».

\section{En « mission »}

Ces intellectuels affirment être demeurés en RDA, car ils avaient une mission à y accomplir au nom du peuple: «On nous reproche à l'Ouest d'être restés en RDA. Nous savions pourtant que le rêve était fini depuis 1968. Mais il y a des liens entre mon pays et moi. Rester? Partir? Avec Volker Braun et d'autres, nous nous étions promis de rester tant qu'il resterait un prêtre, un médecin et un écrivain ${ }^{(53)}$.

Christa Wolf informe ici le lecteur que les écrivains embrassaient une fonction déchirante, celle d'offrir au peuple, malgré la désillusion, un espace de liberté : «c'est au nom $\mathrm{du}$ paradis à venir que nous renoncions à notre vie de tous les jours » ${ }^{(54)}$. On retrouve encore une fois le thème de l'intellectuel victime, qui a toutefois assez de courage pour demeurer dans l'État oppressif, à la faveur du peuple. Wolf déclarera d'ailleurs en 1996 que c'est parce que des lecteurs le lui ont demandé qu'elle est restée en $\mathrm{RDA}^{(55)}$. Elle souligne aussi que, chez elle, « les rêvasseries humanistes avaient remplacé le point de

50 Les initiateurs de Für unser Land avaient appelé la population à signer le manifeste. Voir à ce sujet Frank Thomas Grub, Wende und Einheit im Spiegel der deutschsprachigen Literatur, Berlin, Walter de Guyter, 2003, p. 154.

51 Christoph Hein, "L'exploration du marécage », in: N. Bary, Chronique d'un automne allemand (note 39), p. 177; Christoph Heın, cité dans « Un observateur des modifications », l'Humanité, 2 juin 1995.

Cité dans B. PÄTzold, « Heiner Müller: la mort d’un homme de théâtre » (note 38).

Christa Wolf, dans Jean-Paul LÉONARDINI, « Le courage de la mémoire ", l'Humanité, 14 septembre 1990.

54 Citée dans ibid.

55 B. PARRY, «Christa Wolf: ils ne m'auront pas » (note 23). 
vue de classe ${ }^{(56)}$. De ce fait, Christa Wolf affirme avoir avant tout cherché à protéger la population, puisque c'était son devoir «d'humaniste ». Christoph Hein abonde dans le même sens:

"Nous [les écrivains] devrions pousser l'État à ce débat public, à ce dialogue. [...] nous qui sommes les intellectuels de ce pays, nous aurons un jour à répondre aux questions suivantes: où étiez-vous à l'époque? Où avez-vous pris la parole, pour aussi impuissante qu'elle fût? Aucune réponse ne nous sauvera de la honte si nous continuons aujourd'hui à nous taire [...]. Nous voulons précisément rester pour transformer et améliorer cette société » ${ }^{(57)}$.

La tâche de l'intelligentsia est ainsi présentée comme extrêmement lourde à porter. Cependant, on a l'impression, à la lecture de cette déclaration de Hein, qu'il est aussi intéressé à protéger son identité et son image en tant qu'intellectuel.

Toujours dans le but de construire une image positive des écrivains est-allemands, l'organe culturel du Parti communiste français, Révolution, affirme en novembre 1989: "On ne saurait sous-estimer le rôle de ces guides parfois anonymes, parfois publics, qu'ont été les intellectuels [...]. Si on les a souvent écoutés, c'est qu'ils avaient fait la preuve de leur intégrité morale, de leur courage et de leur clairvoyance " ${ }^{(58)}$. On se soucie peu ici de l'action citoyenne et des premières manifestations du peuple à Leipzig, où les intellectuels ont joué un rôle pratiquement nul. Le dramaturge Volker Braun insiste d'ailleurs particulièrement sur cette supposée fonction de représentant du peuple lorsqu'il affirme croire « que la population n'abandonnera jamais ses acquis. [...] Ce qui l'intéresse, ce n'est pas la liberté floue dans l'autre Allemagne, mais la démocratie concrète en RDA », puis " que cette population est peut-être en condition de se lancer sur ce chemin difficile et expérimental et de tenter autre chose que les attraits de la société de consommation ${ }^{(59)}$. Braun utilise la population comme un miroir de ses propres revendications en lui mettant ses mots en bouche. Ainsi, en se positionnant de cette façon comme la voix du peuple dans la révolution, les écrivains ici étudiés semblent affirmer qu'ils savaient mieux que la population ce qui était bon pour elle. Stefan Heym, candidat en 1994 pour le PDS, parti successeur du SED dans l'Allemagne unifiée, déclare: «Actuellement, une grande insatisfaction règne [en Allemagne], les gens veulent du changement. La question est de savoir s'ils vont s'orienter dans la bonne direction, et non à droite vers le fascisme " ${ }^{(60)}$. L'intellectuel missionnaire doit donc orienter le peuple, le contrôler, pour éviter qu'il ne soit englouti par la droite et par l'Ouest capitaliste.

On relève ainsi un autre paradoxe dans le discours de l'intelligentsia littéraire. En effet, se proclamer porte-parole des intérêts du peuple semble mal s'accorder avec la tentative de rediriger ces revendications. La population est-allemande réclamait alors une plus grande liberté, mais les écrivains qui prennent la parole ne la leur

56 Ch. Wolf, « Rencontre sur la $3^{\mathrm{e}}$ rue » (note 42), p. 21.

57 Christoph HeIn, «La $5^{\mathrm{e}}$ opération fondamentale », in: N. BARY, Chronique d'un automne allemand (note 39), p. 36.

58 G. BADIA, «Intellectuels de RDA : l'esprit de Rosa » (note 47), p. 39.

59 Cité dans F. Combe, "Rencontre avec Volker Braun» (note 48).

60 Cité dans J.-P. PıÉrot, «Stefan Heym ne renonce pas à son combat » (note 46). 
reconnaissent pas lorsqu'ils affirment que les citoyens ne savent pas réellement ce qui est le mieux pour eux. Par exemple, Volker Braun ne fait pas confiance aux citoyens qu'il affirme pourtant vouloir défendre: «Si très vite, on ne met pas quelque chose de nouveau sur la table, le peuple risque de dévorer et la table et les chaises " ${ }^{(61)}$.

Les écrivains lancent de nombreux appels à continuer l'expérience socialiste. Hein, d'abord, déclare qu'il « espère que nous ne sommes pas devenus assez pusillanimes, ou déçus, [...] dénués de courage, pour laisser tomber notre pays. La société où l'on joue des coudes [...] n'est pas ce que j'espère pour mon pays » ${ }^{(62)}$. De son côté, Wolf implore: « Restez dans votre patrie, restez avec nous. Ce sera une vie difficile, certes, mais utile et stimulante ${ }^{(63)}$. Mais rapidement, voyant que la population se détourne en fait du socialisme, c'est une critique sévère du peuple qui émane des écrivains. Après la chute du Mur, les protestations populaires s'estompent peu à peu, la population considérant vraisemblablement ses revendications acquises par l'ouverture des frontières. Les écrivains, de leur côté, prônaient en fait un révisionnisme appelant au retour du socialisme pur, alors que la dissidence citoyenne s'est constituée autour des demandeurs d'exil et de la volonté de liberté. Après le 9 novembre 1989, ces intellectuels voient donc leurs revendications déçues ${ }^{(64)}$ et leur discours se retourne contre la population qu'ils affirmaient d'abord défendre: Stefan Heym reproche aux citoyens l'exode massif de la deuxième moitié de l'année 1989 en affirmant que la «crise ridicule » de la RDA est due à la "désertion de la population " ${ }^{(65)}$. Ce qu'il dénoncera cependant par-dessus tout, c'est que «beaucoup étaient encore plus intéressés à consommer [...] " ${ }^{(66)}$. Déçus par une population qui n'a pas répondu à leurs attentes, peut-être Heym, Braun, Wolf et Hein auraient-ils trouvé plus simple, comme le proposait Bertolt Brecht au gouvernement en juin 1953 dans son poème cynique, de « dissoudre le peuple et d'en élire un autre ${ }^{(67)}$ ?

\section{Victimes de l'Ouest}

Après la chute du mur de Berlin et dans la marche vers l'unification, les écrivains continuent à réclamer un socialisme réformé et redoutent plus que tout l'incorporation de la RDA dans l'Occident capitaliste. Ils chercheront ainsi encore à développer leur image positive, toujours autour des thèmes de la victimisation et de la dissidence, mais cette fois ils se positionnent non seulement comme des victimes du SED, mais aussi de l'Ouest. Martina Doering écrit: «Je me méfie tout autant des hommes politiques de l'autre côté de la frontière, parachutés ici à la course aux élections et qui prétendent représenter mes intérêts » ${ }^{(68)}$. Puisqu'ils ont mis tant d'énergie à donner un sens à leur

61 Cité dans F. Combe, « Rencontre avec Volker Braun» (note 48).

62 Cité dans Claude Prévost, «L’Est était-il donc si mal connu », l’Humanité, 24 juillet 1990.

63 Citée dans B. PARrY, "Christa Wolf: ils ne m'auront pas » (note 23).

64 Ch. Joppкe, East German Dissidents and the Revolution of 1989 (note 6), p. 150, 160.

65 Stefan Heym, "Peut-on encore sauver la RDA? ", in: N. BARY, Chronique d'un automne allemand (note 39), p. 37.

66 Cité dans J.-P. Piérot, « Stefan Heym ne renonce pas à son combat » (note 46).

67 Bertolt Brecht, « The Solution », in: Poems, New York, Routledge, 1997.

68 M. Doering, «L'adieu à un pays » (note 43), p. 6. 
existence socialiste, le capitalisme de l'Ouest ne peut être acceptable: c'est ce que Konrad Jarausch nomme la Trotzidentität (identité d'opposition). Cet historien soutient que les écrivains de RDA ont développé une identité défensive après l'unification qui les a marginalisés. En effet, ils se retrouvent alors sans fonction sociale, doivent à présent compétitionner avec les best-sellers occidentaux et perdent les privilèges que le socialisme leur offrait.

Les écrivains se présentent donc comme des victimes de la RFA, État qui n'offre pas d'aide à la culture. Thomas Brasch, écrivain exilé avant la disparition de la RDA, déclare en ce sens qu'il a « appris à l'Ouest que la littérature peut circuler comme une marchandise " ${ }^{(69)}$ et Heiner Müller laisse entendre qu'il n’a plus été inspiré à créer après l'unification ${ }^{(70)}$. L'Ouest est donc présenté comme le responsable des problèmes professionnels rencontrés par les écrivains dans l'Allemagne unifiée, d'autant plus que les capitalistes auraient lancé une chasse aux sorcières contre les auteurs de l'ex-RDA, comme nous en informe le romancier Hans Joachim Nauschütz au sujet de la controverse suscitée par Was bleibt: "En s'en prenant à Christa Wolf, les grands médias allemands entendaient susciter une discrimination à l'encontre des auteurs de l'Est et convaincre que rien de spécifique, de valable, d'intellectuellement élevé n'avait été produit de ce côté-là » ${ }^{(71)}$. Ce qu'on peut lire entre les lignes de ces déclarations, c'est que la République démocratique allemande offrait aux intellectuels une position enviable qu'ils perdent lors de l'unification.

Il est intéressant de confronter les textes de ces écrivains soucieux de leur image aux critiques formulées par Wolf Biermann à leur encontre après 1989. Lui-même partisan de l'idéal socialiste, le chansonnier offre après la chute du Mur un portrait moins glorieux de l'élite culturelle demeurée en Allemagne de l'Est, affirmant qu'elle est constituée "d'intellectuels poltrons courageux de la RDA [qui] se retrouvent bredouilles. [...] des profiteurs altruistes du stalinisme, [...] des artistes d'État qui assistent, contraints et forcés, à l'anéantissement de leur État, [...] des apôtres de l'égalité aux privilèges menacés ». La critique de Biermann permet de mettre en lumière les incohérences que l'on retrouve dans les discours des écrivains et l'on s'éloigne alors considérablement de l'image positive qu'ils souhaitaient propager. Biermann soulève aussi que les intellectuels qui appellent en 1989 et 1990 à sauver le socialisme " parlent en fait d'argent ". Ainsi, selon lui, les écrivains regrettent avant tout leur position privilégiée et se soucient en fait peu du bien-être de la population. Il contredit aussi leur image de victime en affirmant qu'il est « chose courante [de voir] l'auteur du crime se présenter comme victime séduite » et qu' "évidemment, on peut considérer la menace de perdre certains privilèges comme de la répression ${ }^{(72)}$.

L'Ouest est aussi porteur, pour les écrivains de RDA, du danger d'un retour au fascisme. Eux qui demeuraient convaincus du mythe fondateur de la République

69 Cité dans Jean-Pierre LÉONARDInI, «Thomas Brasch, poète d'Allemagnes », l’Humanité, 6 novembre 1985, p. 21.

70 B. PÄTzold, « Heiner Müller: la mort d'un homme de théâtre » (note 38).

71 Cité dans Patrick Apel-Müller, «Un grand travail sur la mémoire », l’Humanité, 27 octobre 1993.

72 Ces citations proviennent de Wolf Biermann, Seul celui qui change peut rester fidèle à lui-même, La Tour d'Aigues, 1991, p. 8, 10-11, 13, 31. 
démocratique allemande antifasciste considéraient que la RFA ne s'était pas défaite de son passé nazi. Selon Stefan Heym, «la stabilité de l'Europe [était] fondée sur l'existence de deux États allemands » ${ }^{(73)}$, permettant d'éviter de voir renaître la puissance extrémiste de l'Allemagne. Cela permet à Thomas Brasch d'affirmer qu'il « [a] découvert en pratique la perpétuation possible à l'Ouest des traditions qui ont conduit au fascisme et peuvent y ramener. À l'inverse de l'Est, aucun débat utopique n'est possible à l'Ouest. Le socialisme est digne d'amélioration; le capitalisme est tout juste bon à être évacué » ${ }^{(74)}$. D'ailleurs, pour parler du projet d'unification, ces auteurs utilisent des termes associés au nazisme, tel qu'Anschluss (annexion) ${ }^{(75)}$.

Ainsi, le discours formulé par l'intelligentsia littéraire dès septembre 1989 semble avoir été produit avant tout dans le but de créer une image positive des écrivains en fonction de la contestation citoyenne. Puisque cette dernière réclamait le droit à la liberté de parole, à la liberté de circulation et à la démocratie, les écrivains ont développé une présentation d'eux-mêmes dans laquelle ils se posent en victimes de l'oppression du SED et en dissidents. Cela leur permet de s'octroyer le droit de parler au nom du peuple.

L'intelligentsia littéraire insiste donc, au cours de l'automne 1989, sur les positions critiques qu'elle a tenues par le passé et sur son image de "missionnaire " humaniste et défenseur de la population. Cependant, lorsqu'on y regarde de plus près, on comprend que les revendications formulées par les écrivains étaient en fait bien loin de celles réclamées par le peuple. Jusqu'au 9 novembre, ils arrivent somme toute à conserver un certain ascendant sur les citoyens est-allemands puisque la contestation demeure maintenue à l'intérieur de l'État. De ce fait, la manifestation qu'ils organisent le 4 novembre 1989 est un franc succès et plusieurs centaines de milliers de personnes y participent ${ }^{(76)}$. Toutefois, dès l'ouverture du Mur, la mobilisation populaire chute, puisque la liberté réclamée est obtenue. Mécontents, les écrivains développeront un nouveau discours, dans lequel la faute est mise à la fois sur le régime, avec qui ils continuent cependant à collaborer dans une certaine mesure, et surtout sur le peuple « traître » qui se laisse attirer par la superficialité du capitalisme.

Une lecture plus exhaustive du discours de l'intelligentsia littéraire permet aussi d'accéder à la raison qui semble avoir motivé leur refus de voir disparaître l'État socialiste. Outre le fait qu'ils jouissaient de privilèges non négligeables en Allemagne de l'Est, ces écrivains s'étaient construit une identité cohérente avec l'utopie communiste, fortement liée à un antinazisme culpabilisant. Ainsi, il était impossible de rejeter ce travail fait sur eux-mêmes au cours des quarante dernières années, c'est-à-dire pendant le règne du SED en RDA. C'est ce que semble d'ailleurs exprimer Christa Wolf lorsqu'elle demande: "Pourquoi irions-nous perdre collectivement la tête, renoncer à nous-mêmes, à notre histoire [...] et aussi à notre expérience, passée par tant

73 Stefan Heym, «Mercredi des Cendres en RDA », in: N. Bary, Chronique d'un automne allemand (note 39), p. 171.

74 Cité dans J.-P. LÉONARDinI, « Thomas Brasch, poète d'Allemagnes » (note 69).

75 Max von der Grün, cité dans Pierre Duran, «Culture avilie et néonazisme », l'Humanité, 17 février 1990. L'auteur utilise Anschluss pour parler de l'unification des deux Allemagnes. 
d'épreuves [...] " ${ }^{(77)}$. Christoph Hein abonde dans le même sens lorsqu'il écrit: "Pendant quarante années, nous n'avons pas manqué de raisons de désespérer. Ne désespérons pas à la vue du marécage [...], s'il a pu apparaître à nos yeux, il faut y voir un signe de notre succès " ${ }^{(78)}$. Enfin, Bärbel Bohley, membre fondatrice de Neues Forum, adopte une position semblable qu'elle tente même d'appliquer à toute la population, ce qui lui permet d'affirmer que « les Allemands de l'Est ne veulent pas se débarrasser de quarante ans d'histoire [...] " ${ }^{(79)}$. Et c'est cela qui explique le discours développé par les écrivains les mieux établis et les plus reconnus de la République démocratique allemande dès l'automne 1989.

\title{
Résumé
}

À l'automne 1989, alors qu'une vague de protestation populaire déferle sur la République démocratique allemande (RDA), l'intelligentsia littéraire de ce pays s'implique dans les manifestations populaires plus par l'écrit que par l'action. Se proclamant tour à tour victime du régime, dissidente et porte-parole de la population en colère, elle développe cependant un discours qui se rapproche plus de celui de l'État-Parti que de celui des citoyens. Cet article propose une lecture critique des textes et déclarations des écrivains de RDA dans la foulée de la chute du mur de Berlin, en prenant soin de relever les ambiguïtés qu'ils ont alors passées sous silence.

\begin{abstract}
In fall 1989, when a wave of popular protest sweeps over the German Democratic Republic (GDR), the East German writers get involved more by writing than by acting. They describe themselves as victims, dissidents and representatives of an angry population, but develop nevertheless a discourse that is similar to the one of the State and which designates the citizens as " traitors" and "bad socialists". This article offers a reading of the texts and declarations of the East German writers in the wake of the collapse of the Berlin Wall and addresses the ambiguities they then ignored.
\end{abstract}

77 Claude Prévost, « Ces deux livres [...] », l’Humanité, 7 août 1990.

78 Ch. Hein, « L’exploration du marécage» (note 51), p. 179.

79 Citée dans Claude Cabanes, «Éditorial: vu de Berlin », l'Humanité, 13 novembre 1989, p. 3. Neues Forum est un parti politique créé en 1989, dans la foulée des protestations, et qui rassemble de nombreux intellectuels. Ce parti réclame des réformes et non pas l'abandon du socialisme ou l'unification allemande. 